1071

\section{OBESITY AND METABOLIC SYNDROME IN CHILDREN}

\author{
G. Poryadina, M.Y. Scherbakova
}

The Russian State Medical University, Moscow, Russia

Background and aims: among obese children to determine the group of children under a risk of developing metabolic syndrome.

Methods: anthropometry, examination and testing of the children: hormone profile, biochemical blood test, standard glucose tolerance test, questioning of the parents.

Results: 109 overweight children aged from 6 to 17 were examined. The complications are the following: the decrease of High Density Proteins HDL predominates $(22 \%)$, it is followed by the increase of cholesterol (12\%) and high blood pressure (14\%), then follows increase of Low Density Lipoproteins LDL $(7 \%)$ and of level of basal insulin in blood $(9,5 \%)$ and on the last place-Glucose Tolerance Dysfunction $(9,2 \%)$ and increase of uric acid $(4 \%)$. Hard fat and easily assimilated cabohydrates predominated in the diet of most of these children and they lacked vegetables.

Conclusions: Among the children aged from 6 to 17 , who were examined, $21 \%$ have metabolic syndrome. 28 \%have 1 comlplication, typical for metabolic syndrome. The duration of obesity is from 3,3 to 6,4 years - it is longer in the group with metabolic syndrome. The lowest level of physical activity is observed in the group of children with metabolic syndrome $(36,4 \%$ of children don't even attend physical training classes at school. Apparently, genetic predisposition together with lack of exercise and unbalanced, high calory diet increases the number of population with obesity and it's main complication- the metabolic syndrome.

\section{THE OBJECTIVE MEASURE OF SLEEP PATTERN AND ITS ASSOCIATION WITH BODY WEIGHT STATUS IN PRIMARY SCHOOL CHILDREN LIVING IN TEHRAN}

A. Saber Gharamaleki, A. Atoof, M. Hosseini, E. Rajaei, K. Djafarian

Tehran University of Medical Sciences, Tehran, Iran

Objective: To investigate the pattern of sleep and its relationship with weight status in primary school children.

Methods: We carried out a cross-sectional study. Data were obtained from a representative sample of 72 primary school children (30 boys and 42 girls) aged 6-9 years. Sleep pattern was measured for 7 consecutive days using Actigraph accelerometer as well as daily logs. Directly measured height and weight data were used to define overweight and obesity according to the Centers for Disease Control and Prevention's (CDC) 2000 Growth Charts and its recommended cut-off values of 85th and 95th BMI percentile.

Results: No significant difference ( $p>0.05)$ was found in current study between boys and girls for body weight, BMI percentiles, and weekend sleep duration. But girls were found to have higher values than those of boys for Height $(F=7.34, P=0.008)$, age $(F=24.32, P<0.001)$ and lower values for 7 days $(F=10.43, P=0.002)$ and weekdays $(F=9.49, P=$ 0.003 ) sleep duration after adjusting for age. After adjusting the effects of gender and age, average sleep duration among overweight and obese children during weekdays was significantly lower than normal weight children $(\mathrm{F}=3.35, \mathrm{P}<0.05)$. However, we could not find such an association for weekend sleep duration ( $p>0.05)$.

Conclusions: The current study revealed a higher duration of sleep during weekdays among overweight and obese children compared to normal weight one. However, longitudinal design studies are needed to understand the cause and effect relationships between weight status and sleep duration. 